CASO CLÍNICO

\title{
Displasia cortical focal, causa frecuente de epilepsia
}

\section{Focal cortical dysplasia. Case report and literature review}

\section{Clara A. Vidaurre-Herrera* y Laura Quiroz-Rojas}

Departamento de Resonancia Magnética, Grupo CT Scanner, Ciudad de México, México

\section{RESUMEN}

La displasia cortical focal engloba un espectro de trastornos en la organización neuronal y un importante porcentaje de ellos será farmacorresistente. La resonancia magnética es la modalidad de elección para evaluarla, aunque sus características radiológicas pueden ser sutiles y en muchos casos normales. El éxito del tratamiento se relaciona directamente con la capacidad de definir y resecar la zona epileptogénica, por esto se requiere un protocolo optimizado, así como el conocimiento de sus características.

Palabras clave: Displasia cortical focal. Epilepsia. Resonancia magnética.

\section{ABSTRACT}

Focal cortical dysplasia encompasses a spectrum of neuronal organization disorders and a significant percentage of these will be drug-resistant. Magnetic resonance is the modality of choice to evaluate, although its radiological characteristics can be subtle and in many cases normal. The success of the treatment is directly related to the ability to define and resect the epileptogenic zone, therefore its assesment requires an optimized protocol as well as knowledge of its characteristics.

Key words: Focal cortical dysplasia. Epilepsy. Magnetic resonance. 


\section{INTRODUCCIÓN}

La displasia cortical focal (DCF) engloba un espectro de trastornos en la organización neuronal y es la causa de hasta el $25 \%$ de las crisis epilépticas parciales refractarias a tratamiento. Es, por esto, que debemos conocer sus características, así como los protocolos de imagen óptimos para su adecuado diagnóstico y caracterización. La Liga Internacional contra de la Epilepsia (ILAE), en el sistema de clasificación de las displasias corticales, la clasifica como una displasia tipo I.

\section{CASO CLÍNICO}

Paciente masculino de 52 años en estudio por cuadros de crisis convulsivas parciales complejas, sin aura, con mala respuesta al tratamiento, los cuales se inician 1 año antes. Como causa probable se menciona un cuadro de encefalitis viral. Durante la evaluación inicial, se realiza un estudio de tomografía simple, extrainstitucional, la cual reporta pérdida de la diferenciación sustancia gris-blanca en la región temporal izquierda, que se atribuye al proceso inflamatorio antes mencionado. El electroencefalograma (EEG) reveló que el foco epileptógeno se encontraba en el lóbulo temporal.

Es referido a nuestra institución para la realización de una resonancia magnética (RM) contrastada en donde se encuentra un patrón anormal de giros y surcos cerebrales, engrosamiento de la corteza, borrosidad de la unión sustancia gris-blanca e incremento de la intensidad de señal en T2 y FLAIR, localizada en el lóbulo temporal y el hipocampo izquierdos, sin restricción en difusión ni realce patológico tras la administración del medio de contraste (Figs. 1 y 2). Adicionalmente se realizó espectroscopia (Fig. 3), en la cual no se observaron alteraciones, e imágenes por tensor de difusión (Fig. 4), donde se evidenció reducción en la fracción de anisotropía en el lado izquierdo al compararlo con el contralateral. A pesar de los hallazgos altamente sugestivos de DCF, el paciente fue llevado a biopsia.

El reporte histopatológico informó la presencia de áreas de heterotopia neuronal con marcada pérdida tangencial de la laminación. Algunas de las neuronas mostraban nucleomegalia y citoplasma escaso con distribución irregular en medio de focos de proliferación astrocitaria (gliosis). Se concluyó: DCF Ib, heterotopia neuronal focal y gliosis. No se identificaron lesión neoplásica, microorganismos, ni respuesta tisular que lo sugiera en el tejido enviado.

\section{DISCUSIÓN}

La DCF fue descrita por primera vez en 1971 por Taylor, et al., quienes reportaron 10 casos en una serie de 300 lobectomías ${ }^{1}$. Actualmente se reconoce cada vez más en pacientes con epilepsia debido a las mejoras en la adquisición de imágenes de RM, así como en el posprocesamiento de conjuntos de datos en 3D. Representa del 20 al 25\% de las causas de epilepsia focal. De estos, aproximadamente el $76 \%$, será farmacorresistente ${ }^{2}$.

Desde que Palmini en 2004 propuso una clasificación para las DCF ha habido una serie de modificaciones de la misma. En la actualidad, la ILAE ha aceptado la propuesta por Blumcke, 

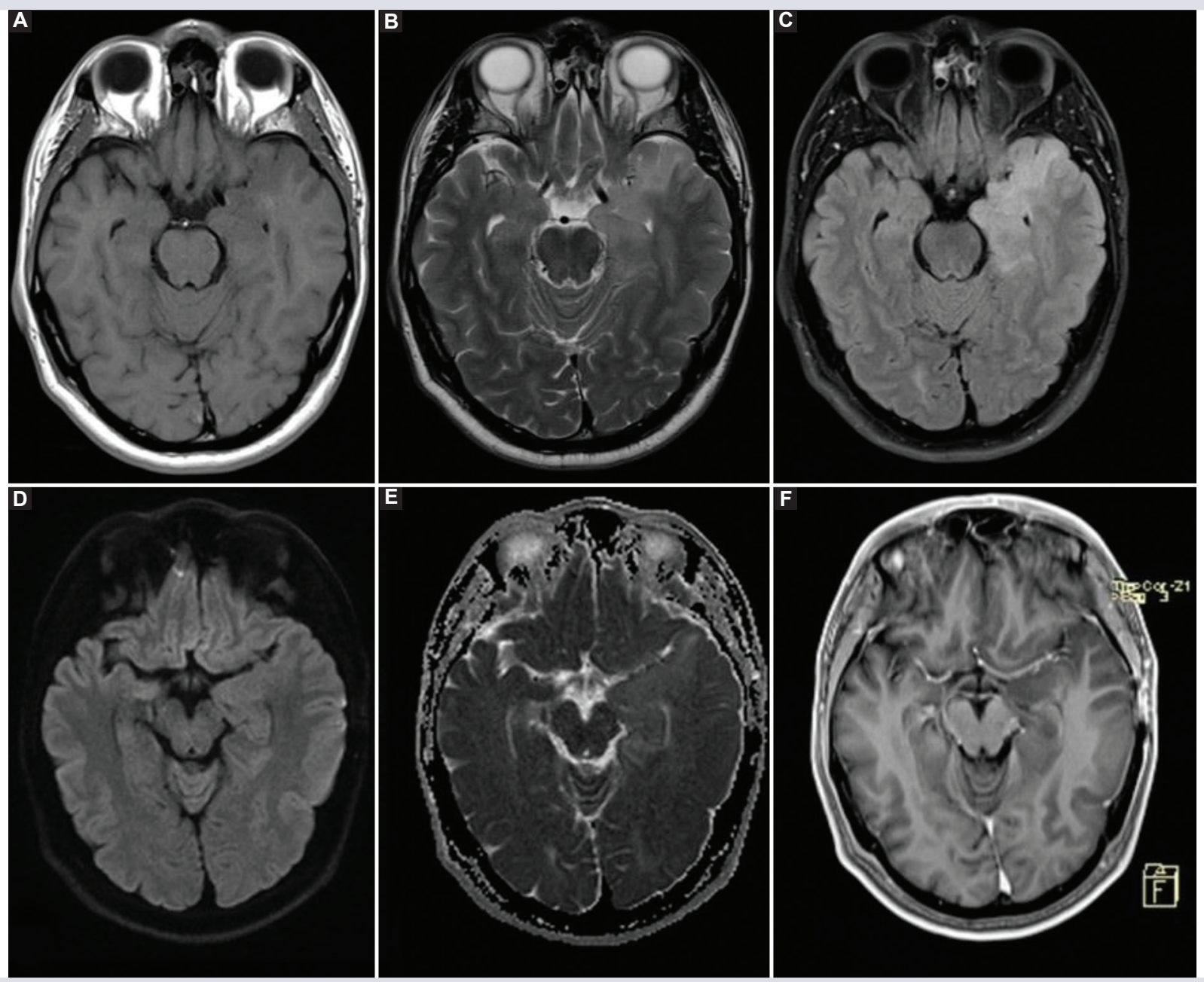

Figura 1. Resonancia magnética en plano axial potenciada. A: T1. B: T2. C: FLAIR. Se observa un patrón anormal de los giros, con engrosamiento cortical, pérdida de la diferenciación de la unión sustancia gris-blanca e incremento en la intensidad de señal en T2 y FLAIR en el lóbulo temporal e hipocampo izquierdos. D-E: imagen de difusión y ADC sin alteración. F: tras la administración del medio de contraste endovenoso no se observó realce.

et al., de $2011^{3}$, que clasifica la DCF en tres tipos y estos a su vez en subtipos. El tipo I se caracteriza por dislaminación y organización interrumpida de la arquitectura tisular, con neuronas y células gliales morfológicamente normales: el tipo Ia con alteraciones radiales, el Ib con laminación horizontal defectuosa y el Ic es una combinación de los anteriores. El tipo II se caracteriza por neuronas megalocíticas displásicas mezcladas con neuronas normales: en el IIa con neuronas dismórficas y en el IIb con presencia de neuronas dismórficas y células en globo. El tipo III es similar al tipo I, pero adyacente a otra lesión: si la lesión adyacente es esclerosis del hipocampo, será IIIa, si es un tumor glial, será IIIb, en caso de malformaciones vasculares será IIIc, y en caso de otra lesión adquirida será IIId. 

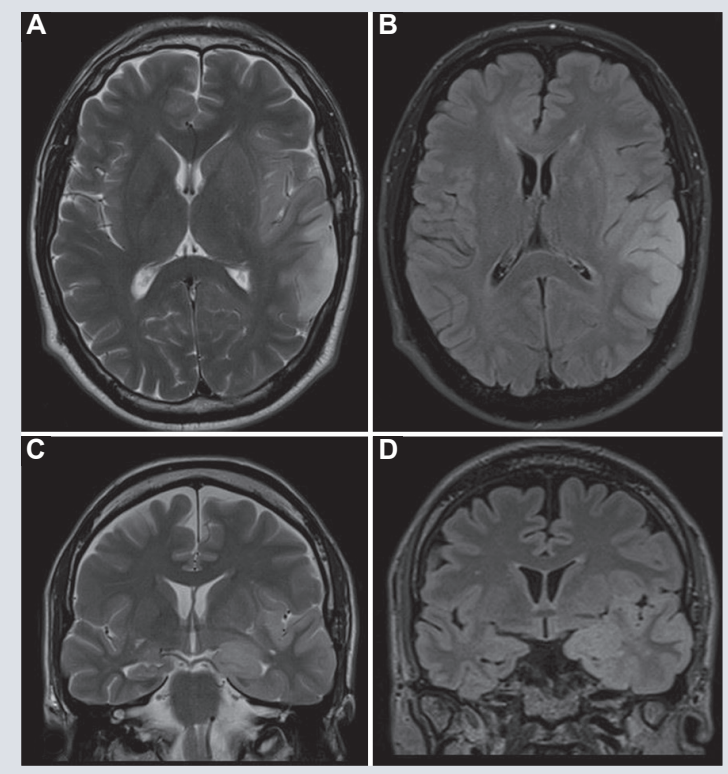

Figura 2. Imágenes de resonancia magnética en un plano superior al de la figura 1, potenciadas. A: en T2. B: FLAIR en el plano coronal. C: en T2. D: FLAIR donde la alteración en el patrón de los surcos y giros, así como el incremento difuso en la intensidad se extiende a la ínsula.

El inicio de la epilepsia ocurre en los primeros años de vida (92.5\% antes de los 16 años) $\mathrm{y}$ es poco frecuente en edades mayores ${ }^{4}$, sin embargo, pueden existir periodos prolongados de tiempo sin convulsiones clínicamente manifiestas, como en el caso de nuestro paciente. Las manifestaciones tardías se han asociado a la falta de anomalías citoarquitectónicas (displasia tipo 1a)².

Las características clínicas en adultos son poco conocidas, aunque se han descrito síntomas de epilepsia del lóbulo temporal. Los pacientes con DCF tipo II son más jóvenes en el momento de la presentación al compararlos con los de tipo $\mathrm{I}^{2}$, y tienen una alta frecuencia de crisis diarias que pueden llegar al estatus epiléptico, además de una localización extratemporal, que afecta principalmente al lóbulo frontal ${ }^{5}$.
La DCF muestra una alta frecuencia de patología dual, la más usual: la esclerosis del hipocampo (68\%). Entre los factores de riesgo se incluyeron convulsiones febriles (16\%), traumatismo craneal $(16 \%)$, infecciones del sistema nervioso central (SNC) (11\%) y accidente cerebrovascular perinatal $(4 \%)^{6}$.

Antiguamente, el diagnóstico de DCF dependía únicamente de los hallazgos patológicos, como consecuencia del resultado de la lobectomía quirúrgica para las convulsiones intratables. Con el advenimiento de nuevas técnicas, se pueden reconocer lesiones atípicas discretas antes de la operación.

La RM es la modalidad de elección para evaluar a los pacientes con posible DCF, sin embargo, en muchos casos no es evidente ninguna anomalía. Algunas características son'1:

- Tipo I: Hipoplasia/atrofia lobular o segmentaria significativa, incremento de la señal en T2 y FLAIR, y disminución en T1, leve borrosidad de la unión sustancia grisblanca y patrón anormal de los giros y surcos. La localización más frecuente es el lóbulo temporal con atrofia del hipocampo.

- Tipo II: Engrosamiento cortical, con ligero incremento de la señal de sustancia blanca en T2, y FLAIR, y disminución en T1, alteración de la intensidad de señal de la sustancia blanca que se extiende hacia el ventrículo (signo del transmanto), borrosidad de la unión sustancia gris-blanca más evidente que en el tipo I, surcos y giros anormales. La localización más frecuente es la frontal y hasta en un tercio de los casos puede ser negativo.

- Tipo III: Comparte las características típicas de las lesiones asociadas 

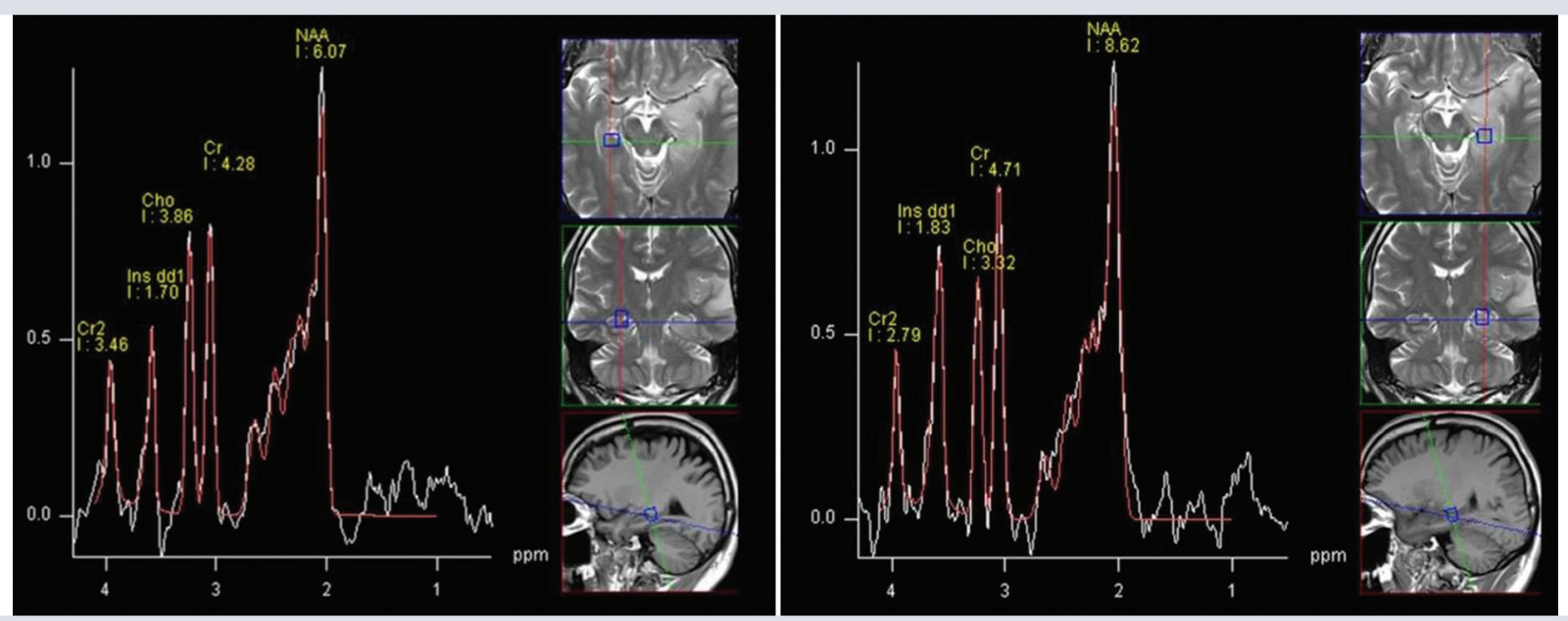

Figura 3. En la espectroscopia comparativa de los hipocampos se observa adecuada concentración y relación entre los distintos metabolitos. A: lado derecho. B: lado izquierdo.
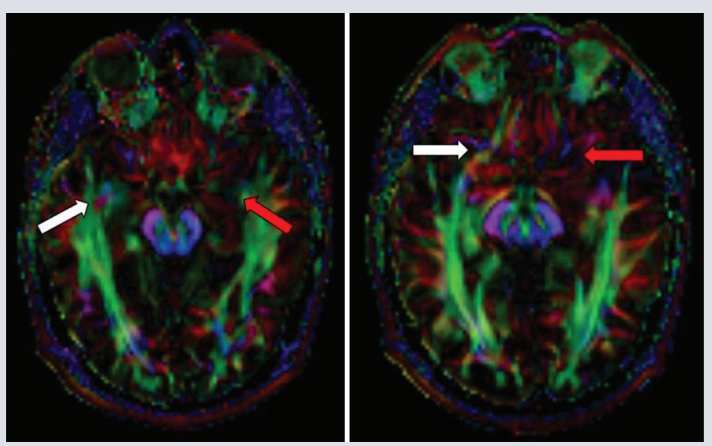

Figura 4. En las imágenes con tensor de difusión se observó reducción en la fracción de anisotropía en el lado izquierdo (flechas rojas) al compararlo con el derecho (flechas blancas).

El éxito de la cirugía de epilepsia se relaciona directamente con la capacidad de definir y, posteriormente, resecar la zona epileptogénica. El uso de resonadores de alto campo magnético (3T) ha reclasificado aproximadamente el $5 \%$ de los pacientes diagnosticados previamente como criptogénicos. La detección de lesiones requiere un protocolo optimizado; se sugiere seguir las siguientes recomendaciones:

- Resonador de alto campo (3 T) cuando esté disponible, para una mejor resolución de contraste de la unión de materia grisblanca.

- Obtener imágenes en T2 (SPACE) coronal oblicua de alta resolución (grosor de corte de $2 \mathrm{~mm}$ ), perpendicular al eje largo del hipocampo. Esto ayuda a la mejor detección de anomalías corticales sutiles. La adquisición de imágenes en FLAIR coronal (grosor de corte de $3 \mathrm{~mm}$ ) confirma las anomalías vistas en T2 a nivel del hipocampo, cortical y subcortical.

- La secuencia de inversión recuperación, 3D coronal, representa mejor las anormalidades focales sutiles, el engrosamiento cortical y el borramiento de la interfaz de la sustancia gris-blanca.

- Imágenes 3D isotrópicas de $1 \mathrm{~mm}$ potenciadas en T1 (MPRAGE o SPGR en el plano axial) y $\mathrm{T} 2$ pueden reformatearse y 
examinarse en múltiples planos para dilucidar malformaciones sutiles.

- Las supresión simultánea de sustancia blanca y líquido cefalorraquídeo (LCR) con secuencias de doble inversión recuperación, permite una mejor valoración de áreas de sustancia gris.

- No se justifica el uso de agentes de contraste IV, con excepción de lesiones tumorales conocidas o sospechadas, facomatosis e infección; además de convulsiones de nueva aparición en un adulto.

La espectroscopia no es parte de la evaluación de rutina, sin embargo, en el contexto de crisis convulsivas, EEG sugerente de epilepsia focal y en el caso de que la RM convencional detecte una lesión focal, es útil para la diferenciación entre displasia versus lesión tumoral. Además se informó que el N-acetil aspartato (NAA) bajo fue la única diferencia estadísticamente significativa entre un grupo con FCD y los sujetos de control de la misma edad. No existe una diferencia estadísticamente significativa en los niveles de colina $(\mathrm{Cho})^{8}$. A pesar de lo anterior, en nuestro caso no existió disminución en la relación NAA/Cr (creatina) o NAA/Cho, y los niveles de colina se encontraron dentro de lo normal.

Las imágenes de tensor de difusión (ITD) muestran cambios microestructurales en la sustancia blanca subcortical adyacente a la anomalía visible de la RM y en los tractos de materia blanca que se proyectan hacia y desde la DCF. Estos cambios microestructurales en la zona epileptogénica de DCF se pueden usar para guiar la resección quirúrgica9. La anisotropía disminuida de la materia blanca adyacente a la corteza malformada y un curso aberrante de las principales vías de la fibra debido a la materia blanca displásica son hallazgos comunes. Por tanto, la ITD se puede usar para evaluar la extensión de la DCF, que a menudo parece más grande que la lesión revelada por RM convencional y en pacientes con RM normal ${ }^{1}$.

La tomografía por emisión de positrones con 18F-fluorodeoxiglucosa (PET-FDG) es muy sensible para detectar la DCF, especialmente en casos de RM negativos. La base fisiológica de esta técnica es el metabolismo de la glucosa cortical y el flujo sanguíneo aumentado en el foco epileptogénico durante una crisis, el mismo que disminuye en los periodos postictal e interictal. La revisión retrospectiva de las imágenes de RM después de la obtención de imágenes nucleares puede revelar una anormalidad cortical sutil pasada por alto en la interpretación inicial7. El PETFDG/RM tiene una mayor sensibilidad (hasta 98\%), especialmente en pacientes con DCF tipo I y RM normal ${ }^{1}$.

Las convulsiones epilépticas en la DCF son difíciles de controlar con tratamiento farmacológico y, a menudo, son intratables, por lo que el tratamiento quirúrgico parece ser el siguiente procedimiento terapéutico. Las cirugías más limitadas se realizan en pacientes de edad avanzada, generalmente debido a DCF tipo I, y ubicadas dentro del lóbulo temporal. Los pacientes más jóvenes suelen tener DCF tipo II, con lesiones más extensas y localización extratemporal, predominantemente frontales. En estos casos, la operación incluye lobectomía/o incluso hemisferectomía ${ }^{1}$.

\section{CONCLUSIÓN}

Los estudios de imágenes en pacientes con epilepsia refractaria buscan identificar una alteración estructural relacionada con el tipo 
de crisis, localizar el foco epileptógeno y proponer un diagnóstico etiológico. Todo esto intervendrá en el tratamiento, planificación prequirúrgica y pronóstico. La detección de lesiones requiere un protocolo optimizado, así como la familiaridad del radiólogo con las características de imagen, sin embargo, debemos considerar la posibilidad de DCF en el diagnóstico de todos los adultos con epilepsia focal intratable y neuroimagen normal.

\section{CONFLICTO DE INTERESES}

Los autores declaran no tener ningún conflicto de interés.

\section{RESPONSABILIDADES ÉTICAS}

Protección de personas y animales. Los autores declaran que para esta investigación no se han realizado experimentos en seres humanos ni en animales.

Confidencialidad de los datos. Los autores declaran que han seguido los protocolos de su centro de trabajo sobre la publicación de datos de pacientes.
Derecho a la privacidad y consentimiento informado. Los autores han obtenido el consentimiento informado de los pacientes y/o sujetos referidos en el artículo. Este documento obra en poder del autor de correspondencia.

\section{BIBLIOGRAFÍA}

1. Bae YS, Kang HC, Kim HD, Kim SH. New classification of focal cortical dysplasia: application to practical diagnosis. J Epilepsy Res. 2012;2(2):38-42.

2. Fauser S, Huppertz HJ, Bast T, Strobl K, Pantazis G, Altenmueller DM, et al. Clinical characteristics in focal cortical dysplasia: A retrospective evaluation in a series of 120 patients. Brain. 2006;129(Pt 7):1907-16.

3. Najm IM, Sarnat HB, Blümcke I. Review: The international consensus classification of Focal Cortical Dysplasia - a critical update 2018. Neuropath Appl Neurobio. 2018;44(1):18-31.

4. Wong-Kisiel LC, Blauwblomme T, Ho ML, Boddaert N, Parisi J, Wirrell E, et al. Challenges in managing epilepsy associated with focal cortical dysplasia in children. Epilepsy Res. 2018;145:1-17.

5. Lerner JT, Salamon N, Hauptman JS, Velasco TR, Hemb M, Wu JY, et al. Assessment and surgical outcomes for mild type i and severe type II cortical dysplasia: A critical review and the UCLA experience. Epilepsia. 2009;50(6):1310-35

6. Bautista JF, Foldvary-Schaefer N, Bingaman WE, Lüders HO. Focal cortical dysplasia and intractable epilepsy in adults: Clinical, EEG, imaging, and surgical features. In: Epilepsy Res. 2003;55(1-2):131-6.

7. Friedman E. Epilepsy imaging in adults: Getting it right. AJR Am J of Roentgenol. 2014;203(5):1093-103.

8. Caruso PA, Johnson J, Thibert R, Rapalino O, Rincon S, Ratai EM. The Use of Magnetic Resonance Spectroscopy in the Evaluation of Epilepsy. Neuroimaging Clin N Am. 2013;23(3):407-24.

9. Widjaja E, Zarei Mahmoodabadi S, Otsubo H, Snead OC, Holowka S, Bells S, et al. Subcortical Alterations in Tissue Microstructure Adjacent to Focal Cortical Dysplasia: Detection at Diffusion-Tensor MR Imaging by Using Magnetoencephalographic Dipole Cluster Localization. Radiology. 2009;251(1):206-15. 